\title{
Functional Reinnervation of the Rat Lower Urinary Tract after Cauda Equina Injury and Repair
}

\author{
Thao X. Hoang, ${ }^{1}$ Victor Pikov, ${ }^{2}$ and Leif A. Havton ${ }^{1}$ \\ ${ }^{1}$ Department of Neurology and Brain Research Institute, David Geffen School of Medicine at University of California, Los Angeles, Los Angeles, California \\ 90095, and ${ }^{2}$ Huntington Medical Research Institutes, Pasadena, California 91105
}

Conus medullaris and/or cauda equina forms of spinal cord injury commonly result in a permanent loss of bladder function. Here, we developed a cauda equina injury and repair rodent model to investigate whether surgical implantation of avulsed lumbosacral ventral roots into the spinal cord can promote functional recovery of the lower urinary tract. Adult female rats underwent sham surgery $(n=6)$, bilateral L5-S2 ventral root avulsion (VRA) injury $(n=5)$, or bilateral L5-S2 VRA followed by an acute implantation of the avulsed L6 and $S 1$ ventral roots into the conus medullaris $(n=6)$. At 12 weeks after operation, the avulsed group demonstrated urinary retention, absence of bladder contractions and external urethral sphincter (EUS) electromyographic (EMG) activation during urodynamic recordings, increased bladder size, and retrograde death of autonomic and motoneurons in the spinal cord. In contrast, the implanted group showed reduced urinary retention, return of reflexive bladder voiding contractions coincident with EUS EMG activation, anatomical reinnervation of the EUS demonstrated by retrograde neuronal labeling, normalization of bladder size, and a significant neuroprotection of both autonomic and motoneurons. In addition, a positive correlation between motoneuronal survival and voiding efficiency was observed in the implanted group. Our results show that implantation of avulsed lumbosacral ventral roots into the spinal cord promotes reinnervation of the urinary tract and return of functional micturition reflexes, suggesting that this surgical repair strategy may also be of clinical interest after conus medullaris and cauda equina injuries.

Key words: bladder; autonomic; regeneration; spinal cord; ventral root avulsion; urodynamic

\section{Introduction}

Although people living with a spinal cord injury consider the return of autonomic function to be one of the highest priorities for improving the quality of life (Anderson, 2004), studies on the neurological repair of bladder control have been relatively few. The level and extent of a spinal cord injury dictates the clinical presentation of the bladder dysfunction (Potter, 2006). After a spinal cord injury rostral to the conus medullaris, a neurogenic bladder may develop with bladder hyperactivity and detrusorsphincter dyssynergia, resulting in inefficient voiding (Schalow et al., 1995; Fowler, 1999). In contrast, injury to the conus medullaris/cauda equina results in loss of segmental reflexes and atrophy of external sphincter muscles, causing an impaired urethral closure and an inability to initiate micturition (Pavlakis et al., 1983; Fowler et al., 1984; Beric and Light, 1992; Hoang and Havton, 2006a).

The two main functions of the lower urinary tract (LUT),

Received March 23, 2006; revised June 19, 2006; accepted July 17, 2006

This work was supported by National Institutes of Health-National Institute of Neurological Disorders and Stroke Grant NS042719, The Paralysis Project of America, and Roman Reed Funds for Spinal Cord Injury Research of California. We thank Mr. Brett Franchini and Ms. Elizabeth Warner for excellent technical support. The repeated-measure ANOVA was performed with the assistance of the University of California, Los Angeles (UCLA) Stat/Biomath Consulting Clinic, and the artwork was done by Lynne Olson at UCLA Photographic Services.

Correspondence should be addressed to Dr. Leif A. Havton, Department of Neurology, David Geffen School of Medicine at University of California, Los Angeles, 710 Westwood Plaza, Los Angeles, CA 90095-1769. E-mail: Ihavton@mednet.ucla.edu.

DOI:10.1523/JNEUROSCI.1259-06.2006

Copyright $\odot 2006$ Society for Neuroscience $\quad$ 0270-6474/06/268672-08\$15.00/0 which is comprised of the bladder and urethra, are to store urine without leakage and to expel urine in a coordinated, controlled manner (de Groat, 1998, 2006; Shefchyk, 2002). In the normal adult rat, storage is dependent on the inhibition of parasympathetic action on the smooth bladder muscle (detrusor) and on the sympathetic tonic activation of the internal urethral sphincter for outflow resistance. During micturition, efficient voiding is dependent on synchronous activation of the detrusor for contraction, relaxation of the internal urethral sphincter, and bursting activity of the striated external urethral sphincter (EUS) for enhanced urine flow (Maggi et al., 1986; Kruse et al., 1993). Normal control of bladder function involves a complex interaction between the cerebral cortex, pontine micturition center, sympathetic and parasympathetic nervous systems, and somatic motoneurons in the lumbar spinal cord.

After an avulsion injury of spinal roots, implantation of the avulsed ventral roots into the spinal cord emerged as one surgical approach for reinnervating hindlimb and forelimb muscles (Carlstedt et al., 1986; Hallin et al., 1999; Chai et al., 2000; Gu et al., 2004). Using this strategy, functional reinnervation of mostly proximal skeletal muscles was demonstrated with coactivation of antagonistic muscles in humans (Carlstedt et al., 1995). Because proper control of the LUT is complex and requires coordinated activity between autonomic and somatic motor neurons, it is not known whether urinary function may be recovered by similar implantation of lesioned ventral roots into the spinal cord.

Here, we developed a ventral root avulsion (VRA) and im- 
plantation model of cauda equina injury and repair in adult female rats. To examine the effects of implantation on the LUT function, we monitored urinary retention, performed urodynamic recordings and anatomical retrograde labeling, as well as measured bladder size and weight. Our results show that implantation of avulsed lumbosacral ventral root into the conus medullaris promotes functional and anatomical reinnervation of the LUT.

\section{Materials and Methods}

Animals. All animal procedures were performed according to the standards established by the National Institutes of Health Guide for the Care and Use of Laboratory Animals. The experimental protocols were approved by the Chancellor's Animal Research Committee at University of California, Los Angeles. All animals were housed in a room with a $12 \mathrm{~h}$ light/dark cycle, and they had access to food and water ad libitum. Seventeen adult female Sprague Dawley rats (200-220 g; Charles River Laboratories, Raleigh, NC) were included in the study. The animals were divided into three groups: (1) laminectomy and dura opening (sham rats; $n=6$ ), (2) bilateral L5-S2 VRA (avulsed rats; $n=5$ ), and (3) bilateral L5-S2 VRA and bilateral acute implantation of the L6 and S1 ventral roots into the spinal cord (implanted rats; $n=6$ ).

VRA and implantation surgery. Under general gas anesthesia with $2-2.5 \%$ isoflurane (Abbott Laboratories, North Chicago, IL), a bilateral lumbar laminectomy (L1-L4) and opening of the dura mater were performed in all rats. Anatomical landmarks were used to identify the L5, L6, S1, and S2 ventral roots under a surgical microscope. The sham group did not undergo any additional surgery. For the avulsed and implanted groups, all four ventral roots were bilaterally avulsed to completely abolish all functional contractions of the bladder, a midline organ, mediated by autonomic neurons in the L6 and S1 spinal cord segments, as well as all activity of the EUS, which is directly innervated by dorsolateral (DL) motoneurons located primarily in the L6 segment (Schrøder, 1980; Nadelhaft and Booth, 1984; McKenna and Nadelhaft, 1986; Marson, 1997). By applying constant traction with a pair of fine jeweler's forceps along the normal course of each individual root, all rootlets of each root were separated from the anterior surface of the spinal cord. The avulsed group did not undergo any additional surgery. For the implanted group, the rootlets of the avulsed L6 and S1 ventral roots were trimmed, and two small scalpel incisions of $\sim 0.5 \mathrm{~mm}$ deep were made bilaterally into the lateral funiculus of the L6 and S1 spinal cord segments. The roots were implanted into the spinal cord in their respective incision sites (supplemental Fig. $1 a$, available at www.jneurosci.org as supplemental material). Because the L6 and S1 ventral roots are of small caliber, no tissue glue was necessary to hold the roots in place. An 8-0 suture was loosely tied around each implanted root to allow for easier identification during later dissections. For all animals, a titanium mesh cage was placed over the laminectomy site to stabilize the vertebral column and protect the spinal cord from compression by the overlying muscles (Nieto et al., 2005). The overlying paraspinous muscles and skin were subsequently sutured in layers, and all animals were allowed to recover.

Urine expression and locomotor assessment. Urinary bladders were manually expressed three times per day for $2 \mathrm{~d}$ after surgery, and then twice per day every 12 h ( 9 A.M. and 9 P.M.) until termination of the experiment at 12 weeks after operation. Once per week, a $24 \mathrm{~h}$ urine output was measured by two collections in a weighing boat $12 \mathrm{~h}$ apart and weighed on a scale. In addition, once per week, locomotor behavioral assessment was performed in an open field.

Urodynamic recording. All animals underwent urodynamic recordings at 12 weeks after operation before the termination of the studies. Under general gas anesthesia using $2-2.5 \%$ isoflurane, a catheter (PE-50) was inserted through the urethra into the bladder to measure the intravesical pressure (IVP) of the bladder. The transurethral catheter was connected to a pressure transducer (DataLab 2000, model 70754; Lafayette Instruments, Lafayette, IN). For electromyographic (EMG) recordings, two 50 $\mu \mathrm{m}$ epoxy-coated platinum-iridium wire electrodes (A-M Systems, Everett, WA) were hooked percutaneously into the EUS muscle. For this purpose, a half-inch 30 gauge needle with a hooked electrode at the tip was inserted into the urethra along the side of the catheter into the EUS and withdrawn, leaving the electrodes embedded in the muscle (supplemental Fig. $1 b$, available at www.jneurosci.org as supplemental material). Animals were then placed in a prone position and allowed to acclimate to a decreased concentration of isoflurane, $0.6-0.8 \%$, for $\sim 1 \mathrm{~h}$ before infusion of saline $(13.2 \mathrm{ml} / \mathrm{hr})$ into the bladder through the catheter. The rat was able to expel the urine around the catheter into a dish. The animals were kept only lightly anesthetized, because previous studies have shown that anesthesia significantly reduces voiding efficiency (Yoshiyama et al., 1994; Matsuura and Downie, 2000). The experiment was performed in a quiet room with a cloth cone used to keep the animal in the dark and maintain normal body temperature. One advantage with sedated animals was the minimal EMG artifacts from movement. The recording sessions lasted for $2-3 \mathrm{~h}$ for each animal.

Analysis of urodynamic data. A minimum of $20 \mathrm{~min}$ of recording was analyzed for each animal. The maximum IVP increase during each contraction (calculated by subtracting the peak amplitude from baseline) and duration of contraction were determined. The number of contractions that occurred within a time period was averaged to define the intercontraction interval (ICI). During precontraction, the rise and fall of contraction, and postcontraction, we analyzed the following: (1) EMG duration, (2) EMG amplitude, and (3) EMG firing rate. These measurements allowed us to determine the synchronicity of the bladder and sphincter activity during contraction. Voided volume ( $\mathrm{Vv})$ was estimated by calculating the product of the ICI and the rate of bladder filling (RF). The total volume $(\mathrm{Vt})$ of the bladder was measured after intravascular perfusion of the animal and removal of the bladder at the end of the study and was used to calculate the voiding efficiency, or percentage of bladder volume voided $(\mathrm{Vv} / \mathrm{Vt} \times 100)$.

Tracer injection. After the completion of the urodynamic recordings, the anesthesia level was increased to $2 \%$ for the retrograde labeling procedure. By inserting a 30 gauge $10 \mu \mathrm{l}$ Hamilton syringe needle into the urethra along the side of the catheter, similar to the above procedure for inserting the EMG electrodes, $0.5 \mu \mathrm{l}$ of $2 \%$ Fast Blue (Sigma, St. Louis, $\mathrm{MO}$ ) was injected into four equidistant sites along the circumference of the EUS muscle ( $2 \mu \mathrm{l}$ total). Animals were allowed to recover and survive for another $5 \mathrm{~d}$ to allow for retrograde transport and labeling of spinal cord neurons.

Perfusion and dissection. Animals were anesthetized with Nembutal (100 mg/kg; Abbott Laboratories), their bladders were completely expressed of residual urine, and all rats were intravascularly perfused with $200 \mathrm{ml}$ of phosphate buffer followed by $500 \mathrm{ml}$ of $4 \%$ paraformaldehyde $(\mathrm{pF})$. The spinal cords were dissected under a microscope and closely inspected to ensure the correct spinal cord levels of root avulsion and the structural integrity of the implanted roots. We excluded animals that had dislodged implanted roots or incorrect/incomplete levels of injury and implantation. The lumbosacral portion of the spinal cord was removed, postfixed in $4 \% \mathrm{pF}$ for $24 \mathrm{~h}$, transferred to $30 \%$ sucrose for $24 \mathrm{~h}$, frozen, and cut $(40 \mu \mathrm{m})$ transversely. The bladder was dissected out, blotted dry, and weighed. The bladder was flattened and the perpendicular length (L) and width (W) were measured. The formula for a prolate spheroid $\left[(4 / 3) \times \pi \times \mathrm{L} \times \mathrm{W}^{2}\right]$ was used to estimate the total bladder volume.

Counts of retrogradely labeled neurons. Serial wet sections of the lumbosacral (L5-S2) spinal cord were placed under a Nikon (Tokyo, Japan) E600 light microscope equipped with a fluorescent UV excitation filter. The absolute number of all fluorescent Fast Blue-labeled neurons was counted on both sides of the spinal cord, and the location of each labeled neuron was noted. Images were captured with a Spot RT-slider monochrome camera (Diagnostics Instruments, Sterling Heights, MI) attached to the microscope.

Immunohistochemistry. After the quantitative analysis of the retrogradely labeled neurons, spinal cord sections from the L6 and S1 segments were immunohistochemically processed for choline acetyltransferase (ChAT) to identify surviving preganglionic parasympathetic neurons (PPNs) and motoneurons. Although ChAT expression is downregulated shortly after injury, it returns and reliably labels PPNs and motoneurons by 4 weeks (Rende et al., 1995; Hoang et al., 2003). The sections were first placed in $0.3 \% \mathrm{H}_{2} \mathrm{O}_{2}$ for 10 min to quench endogenous peroxidase activity, rinsed in PBS, and incubated overnight with a goat 
ChAT primary antibody (1:200; Chemicon, Temecula, CA) in 2\% normal horse serum $/ 0.3 \%$ Triton X-100/PBS at room temperature. All sections were then rinsed, placed in biotinylated anti-goat secondary antibody (1:200; Vector Laboratories, Burlingame, CA) for $1 \mathrm{~h}$ at room temperature, rinsed, incubated for $1 \mathrm{~h}$ with avidin-biotin complex (1: 100; Vectastain ABC Elite kit; Vector Laboratories), and then visualized as a light-stable product using diaminobenzidine (Sigma) as the chromogen. Some sections were additionally counterstained with cresyl violet. Primary and secondary antibodies were omitted as negative controls.

Quantification of autonomic and somatic motor neurons. We used a slight modification of the nonbiased physical disector method (Coggeshall and Lekan, 1996; West, 1999) for obtaining stereological counts of the ChAT-immunolabeled neurons of each rat as performed previously in our laboratory (Hoang et al., 2003; Akhavan et al., 2006). Because of the anatomical location of PPNs and the motor nuclei in the L6 and S1 segments, we counted PPNs and ventral (V) motoneurons in the S1 segment. DL and dorsomedial (DM) motoneurons were counted in the L6 segment. Neurons on both sides of the spinal cord were counted in every fourth section ( $n=10$ sections in the S1 segment, and $n=6$ sections in the L6 segment). We counted all neuronal profiles in a section but excluded neurons with cell bodies that were bisected at the rostral surface of each $40 \mu \mathrm{m}$ section. The counts were then multiplied by 4 for an approximate total span of $1.6 \mathrm{~mm}$ ( $\mathrm{S} 1 \mathrm{seg}-$ ment) and $1 \mathrm{~mm}$ (L6 segment).

Statistics. Quantitative data were expressed as mean \pm SE. For bladder and spinal cord morphological analysis as well as urodynamic studies, a one-way ANOVA test was used followed by the Tukey test (SigmaStat 3.1; Systat Software, Point Richmond, CA). For urine collection data, the means were compared using a repeated-measures ANOVA model. The Pearson correlation was used for correlation analysis. The $p$ value was computed from the post hoc $t$ statistics. $p<0.05$ was considered statistically significant.

\section{Results}

\section{Bladder and hindlimb assessments}

Before surgery, all rat bladders were expressed and found to retain no or a minimal amount of residual urine, a finding typical of normal rats. After injury, both avulsed and implanted groups retained more urine than the sham group (Fig. 1) $(p<0.05)$. The average daily residual urine volume retained over 11 weeks was $0.02 \pm 0.01 \mathrm{ml}$ for sham rats $(n=6)$, $3.49 \pm 1.16 \mathrm{ml}$ for avulsed rats $(n=5)$, and $2.16 \pm 0.93 \mathrm{ml}$ for implanted rats $(n=$ $6)$. The amount of urine retention in both the avulsed and implanted groups already at week 1 was less than the typical amount of urine production within $24 \mathrm{~h}$ (Kerns et al., 2000; Krinke, 2000), suggesting the presence of urine leakage. Despite this confounding factor, avulsed rats retained significantly larger amounts of urine compared with sham rats for essentially the entire duration of the study, whereas implanted rats did not retain statistically larger amounts of urine compared with sham rats during the second half of the study.

A bilateral L5-S2 VRA injury resulted in minor movement deficits. All injured rats were weight bearing and locomoted with coordinated hindlimb plantar steps. The only abnormality was a

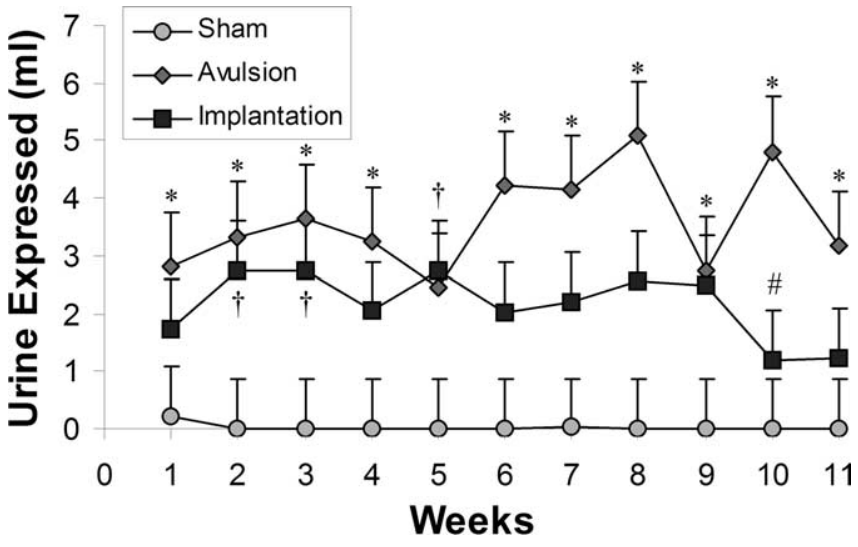

Figure 1. Weekly urine expression measurements demonstrate the amount of urine retained in both injured groups compared with sham controls during the course of 11 weeks after operation. Compared with the sham group, the avulsed group retains significantly increased amounts of urine essentially throughout the study, whereas the implanted group shows significantly increased urine retention only during the first but not second half of the study. ${ }^{*} p<0.05$ between sham and avulsed groups; ${ }^{\dagger} p<0.05$ between sham and implanted groups; ${ }^{\#} p<0.05$ between avulsed and implanted groups.
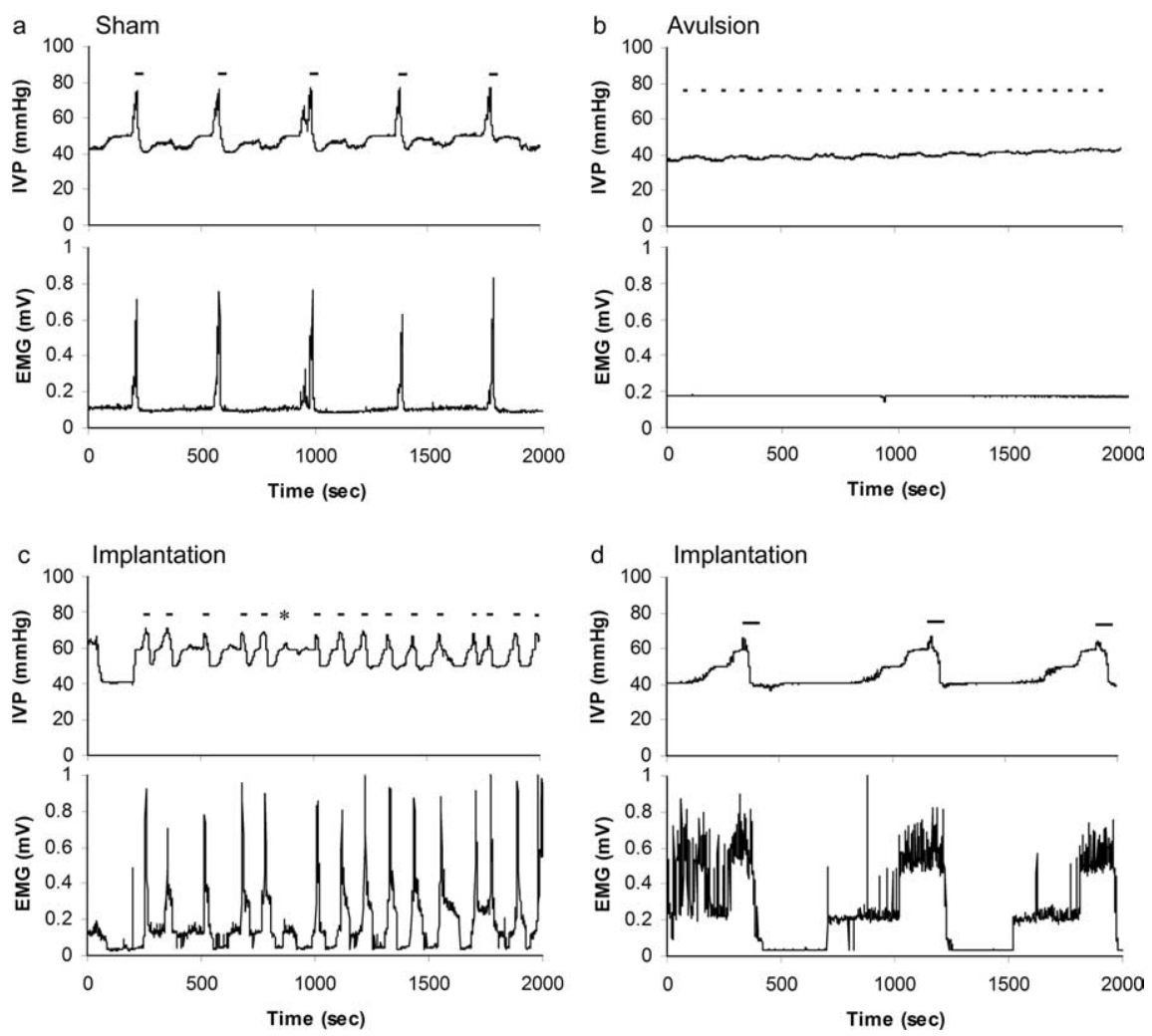

Figure 2. Urodynamic recordings show functional reinnervation of the bladder and EUS muscle at 12 weeks after implantation. The top and bottom graphs are simultaneous recordings ( $~ 33 \mathrm{~min}$ ) of the bladder IVP and rectified EMG activity of the EUS during saline infusion, respectively. Black bars above the pressure spikes indicate voiding periods. $a$, Sham rats show high-frequency EMG activation coincident with reflex bladder contractions and voiding approximately every $350 \mathrm{~s}$ with quiescent filling phases. $\boldsymbol{b}$, After avulsion, no bladder contractions and no EUS EMG activity are observed. Note that the dotted black bars represent the continuous passive dripping of urine once the bladder reaches its volume capacity. After implantation, all animals demonstrate a rise in bladder IVP, coincident EUS EMG activation, and voiding contractions (two examples are shown: $\mathbf{c}$ and $\boldsymbol{d}$ ). $\boldsymbol{c}$, $\boldsymbol{d}$, In some animals, the duration of the IVP and EMG activity as well as intercontraction interval are particularly short (c) or long (d). Nonvoiding contractions are occasionally observed (asterisk).

limited bilateral ankle joint range of active movement with only small improvements during the first 4 weeks after injury. There was no noted difference in mobility between the avulsed and implanted groups, probably because the L5 root, which normally 

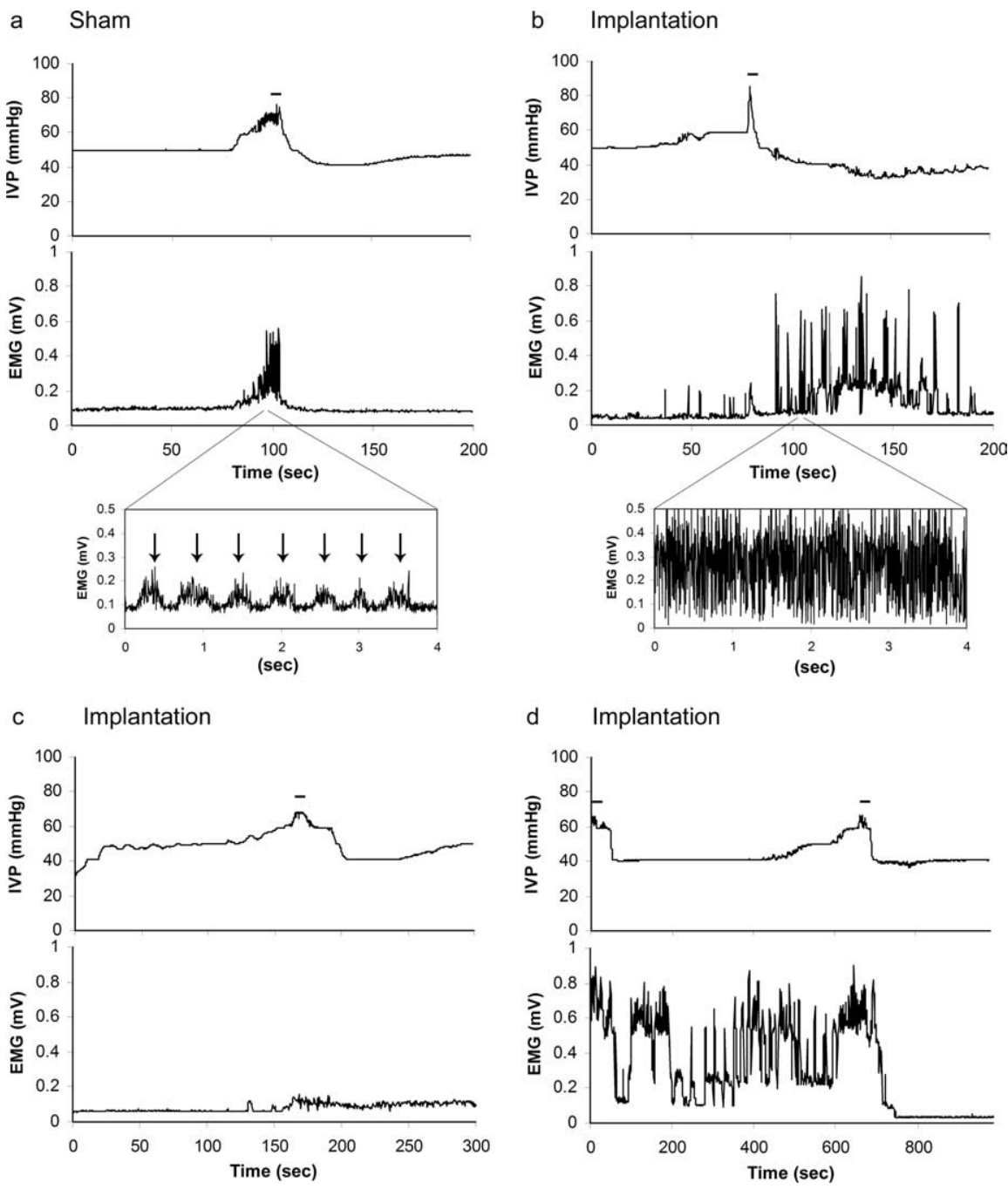

Figure 3. Asynchronous bladder and EUS activities are evident after implantation. $\boldsymbol{a}, 0$ ne cycle of bladder contraction in a sham rat shows a normal rise in IVP coinciding with EUS activation. A shorter timescale shows periodic high-frequency bursting EMG activation (arrows) separated by EMG silence. $\boldsymbol{b}$ - $\boldsymbol{d}$, Juxtaposing the synchronous activity of sham rats are recordings of implanted rats demonstrating examples of delayed EMG activation during contraction (b), contractions without any EMG activation (c), and EMG activation during filling phase ( $\boldsymbol{d}$ ). The shorter timescale in the implanted rat ( $\boldsymbol{b}$, inset) shows lack of intermittent bursting EMG activation. Black bars above the peak of pressures indicate voiding periods. Note that the timescales differ between panels.

provides innervation of select distal hindlimb muscles, was not implanted.

\section{Functional assessment of LUT using urodynamic recordings}

To examine the function of the bladder and EUS muscle, urodynamic recordings were performed at 12 weeks after operation for all studied groups. For sham rats (Fig. 2a), during the bladderfilling phase, there was a low baseline level of bladder IVP and EUS EMG activity. As the infused volume of saline reached the threshold bladder volume of $1.7 \pm 0.4 \mathrm{ml}(n=6)$, there was a sharp increase in bladder IVP, high-frequency EMG activation of the EUS, and voiding occurring simultaneously. At a constant infusion rate, such bladder contractile cycles occurred approximately once every $5 \mathrm{~min}$. In the avulsed group (Fig. 2b), there were no signs of any EUS EMG activation or of any rise in IVP during saline infusion of the bladder. When the bladder volume reached its maximum capacity and distension $(5.5 \pm 2 \mathrm{ml} ; n=5)$, in the absence of any bladder contractions or EUS EMG activation, saline passively leaked from the urethra and dripped steadily into the collecting dish (overflow incontinence). In contrast, in the implanted group (Fig. $2 c, d$ ), there was a wide range of bladder and EUS responses that occurred at a bladder threshold volume of $3.4 \pm 1.2 \mathrm{ml}(n=6)$. For all implanted rats, there were bladder contractions concurrent with sphincter EMG activation and voiding. In some rats, bladder contractions occurred frequently (Fig. $2 c$ ), whereas other animals demonstrated longer periods between contractions (Fig. $3 d)$. Most of the reflex contractions resulted in voiding (Fig. $2 c, d$, black bars). However, in three of the six implanted rats, occasional incidences of weak contractions resulted in nonvoiding contractions (Fig. $2 c$, asterisk). Stepwise recruitment was seen in the EMG activation of some animals (Fig. 2d).

Close examination of each contraction cycle in sham rats showed first a rise in IVP, shortly followed by EUS activation, and finally urine expulsion (Fig. $3 a$ ). For implanted rats, voiding always occurred concurrently with bladder contractions. However, different types of asynchronous activity were observed in the implanted rats (Fig. $3 b-d$ ). In some rats, there was a prolonged or delayed onset of EMG activation lasting beyond the duration of the bladder contraction (Fig. $3 b$ ). Occasionally, bladder contractions were not accompanied by EMG activation (Fig. 3c). Conversely, EMG activation infrequently occurred in the absence of contractions (Fig. $3 d$ ). For sham rats, a shorter timescale analysis (Fig. 3a, inset) of the EMG activation phase showed periodic highfrequency bursting activity separated by periods of quiescence, a process characteristic of normal rat micturition (Mersdorf et al., 1993; Kakizaki and de Groat, 1996; Cheng and de Groat, 2004). In contrast, the high-frequency EMG activation of implanted rats was different compared with sham rats with bursting activity not typically separated by periods of quiescence (Fig. $3 b$, inset).

Quantitative analysis of the urodynamic recordings was performed for all groups (Table 1). The avulsed group was significantly $(p<0.05)$ different from both the sham and implanted groups in all parameters including bladder contractile properties, EUS EMG activation, and voiding efficiency. Although the implanted rats demonstrated an improvement compared with the avulsed rats, the implantation procedure resulted in a significantly $(p<0.05)$ lower maximum IVP and voiding efficiency compared with sham controls.

\section{Effect of reinnervation on bladder size}

To determine the effects of reinnervation on the peripheral end organ, the bladder was measured for volume and weight after perfusion (Fig. 4). The bladder volume of avulsed rats (10.7 \pm 3.3 $\mathrm{ml} ; n=5)$ was significantly larger $(p<0.05)$ than that of both sham $(2.5 \pm 0.2 \mathrm{ml} ; n=6)$ and implanted rats $(3.6 \pm 0.8 \mathrm{ml} ; n=$ $6)$. The bladder weight of avulsed rats $(588 \pm 127 \mathrm{mg})$ was also 
Table 1. Quantitative urodynamic outcomes in the experimental groups

\begin{tabular}{|c|c|c|c|}
\hline & $\begin{array}{l}\text { Sham } \\
(n=6)\end{array}$ & $\begin{array}{l}\text { Avulsion } \\
(n=5)\end{array}$ & $\begin{array}{l}\text { Implantation } \\
(n=6)\end{array}$ \\
\hline \multicolumn{4}{|l|}{ Bladder contractions } \\
\hline Duration (s) & $66 \pm 19$ & $0 \pm 0^{*}$ & $91 \pm 31$ \\
\hline $\operatorname{Max}$ IVP (mmHg) & $27 \pm 2$ & $0 \pm 0^{*}$ & $16 \pm 4^{* *}$ \\
\hline $\mathrm{ICl}(\mathrm{s})$ & $324 \pm 52$ & $0 \pm 0^{*}$ & $309 \pm 68$ \\
\hline \multicolumn{4}{|l|}{ EMG activity of EUS } \\
\hline Mean amplitude (mV) & $0.14 \pm 0.03$ & $0 \pm 0^{*}$ & $0.20 \pm 0.08$ \\
\hline Precontraction & $0.08 \pm 0.01$ & $0 \pm 0^{*}$ & $0.13 \pm 0.05$ \\
\hline Rise & $0.13 \pm 0.03$ & $0 \pm 0^{*}$ & $0.21 \pm 0.08$ \\
\hline Fall & $0.14 \pm 0.02$ & $0 \pm 0^{*}$ & $0.20 \pm 0.08$ \\
\hline Postcontraction & $0.08 \pm 0.02$ & $0 \pm 0^{*}$ & $0.14 \pm 0.03$ \\
\hline Mean firing rate $(\mathrm{Hz})$ & $124 \pm 49$ & $0 \pm 0^{*}$ & $167 \pm 97$ \\
\hline Precontraction & $7 \pm 4$ & $0 \pm 0^{*}$ & $57 \pm 41$ \\
\hline Rise & $121 \pm 47$ & $0 \pm 0^{*}$ & $181 \pm 95$ \\
\hline Fall & $122 \pm 54$ & $0 \pm 0^{*}$ & $168 \pm 100$ \\
\hline Postcontraction & $35 \pm 23$ & $0 \pm 0^{*}$ & $65 \pm 24$ \\
\hline Voiding efficiency (\%) & $51 \pm 6$ & $0 \pm 0^{*}$ & $33 \pm 5^{* *}$ \\
\hline
\end{tabular}

${ }^{*} p<0.05$ compared with both sham and implanted groups; ${ }^{* *} p<0.05$ compared with sham.
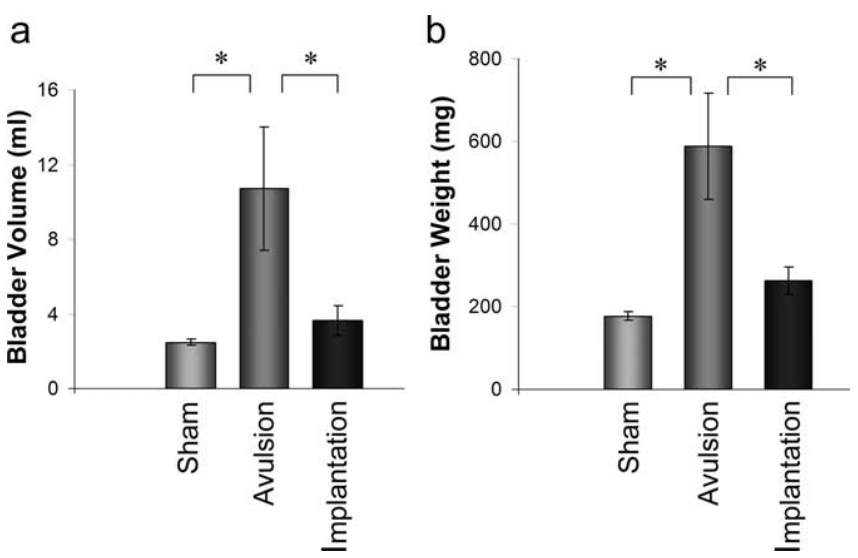

Figure 4. Root implantation promotes normalization of bladder size by 12 weeks after operation. The bladder volume $(\boldsymbol{a})$ and weight $(\boldsymbol{b})$ significantly increase after avulsion compared with sham rats $\left({ }^{*} p<0.05\right)$. After implantation, both bladder volume and weight are significantly reduced compared with avulsed rats $\left({ }^{*} p<0.05\right)$ and returned to normal values with no significant differences between implanted and sham rats.

significantly heavier $(p<0.05)$ than that of both sham $(177 \pm 10$ $\mathrm{mg})$ and implanted rats $(263 \pm 34 \mathrm{mg})$. There was no significant difference in bladder weight or volume between sham and implanted groups.

\section{Retrograde labeling from the EUS muscle}

To confirm anatomical reinnervation of the LUT, Fast Blue was injected into the EUS muscle of all animals. In the sham group, an average of $28 \pm 11$ neurons $(n=6$; Fig. $5 a)$ in the DL motor nucleus were retrogradely labeled, spanning a rostrocaudal distance of $0.8-1.2 \mathrm{~mm}$. In the avulsed group $(n=5)$, no Fast Blue-labeled neurons were observed in any of the animals during a thorough examination. In contrast, in the implanted group, Fast Blue-labeled spinal cord neurons were detected in the DL motor nucleus spanning $0.4-1.0 \mathrm{~mm}$ rostrocaudally ( $7 \pm 5$ neurons; $n=6$ ) (Fig. 5b). No Fast Blue-labeled neurons were found outside of the DL motor nucleus in the implanted group.

\section{Neuroprotection of spinal cord neurons}

The number of surviving PPNs and motoneurons at 12 weeks after operation were counted using ChAT immunohistochemistry. Sections counterstained with cresyl violet confirmed that quantification of motoneurons using ChAT expression was reliable. ChAT-positive neurons were labeled in the intermediolateral nuclei, ventral horn, adjacent to the central canal, and occasionally in the dorsal horn and in lamina VII (Fig. $5 d-f$ ), in agreement with previous studies (Barber et al., 1984). The number of surviving PPNs after implantation was significantly increased compared with the avulsed group but remained significantly decreased compared with the sham group (Table 2) (Fig. $5 g$ ). Similar to PPNs, there was a significant avulsion-induced loss of motoneurons in all motor nuclei of the L6 and S1 segments (DL, DM, and V), but a significant neuroprotective effect was demonstrated with the root implantation (Table 2). There was no significant difference between the percentage of surviving PPNs and motoneurons in either the avulsed or implanted group (Fig. $5 g$ ).

In addition, in the implanted group, there was a significant correlation between the number of surviving motoneurons and voiding efficiency (Fig. 5h). There was no relationship between the number of surviving PPNs and voiding efficiency.

\section{Discussion}

Our results demonstrate that after a bilateral L5-S2 VRA, all parasympathetic and somatic spinal cord outputs for bladder contraction and EUS muscle activation are eliminated, resulting in retrograde death of PPNs and motoneurons, an inability to actively void, and hypertrophy of the bladder. In contrast, acute implantation of the avulsed lumbosacral ventral roots into the conus medullaris resulted in neuroprotection of the efferent spinal cord neurons as well as provided a conduit for reinnervation of the LUT, return of functional micturition reflexes, normalization of bladder size, and improvement in voiding efficiency.

\section{Bladder function in upper versus lower motor neuron injury}

Our lumbosacral VRA injury, as a model for conus medullaris/ cauda equina injuries, results in a lower motor neuron injury with different functional consequences compared with an upper motor neuron injury. For instance, spinal cord injuries at the thoracic region leave the lumbosacral segmental circuitry intact, and such injured rats may regain the ability to reflexively void over several weeks (Kruse et al., 1993; Pikov et al., 1998; Cheng and de Groat, 2004). Furthermore, there is retention of essentially all produced urine (Pikov and Wrathall, 2001; Keirstead et al., 2005). In contrast, we show that rats with a bilateral VRA injury do not reflexively void because of a permanently interrupted segmental reflex. The urine collection data show that although both the avulsed and implanted groups retain increased residual volumes, the collected weights are markedly below the expected weight for normal urine production of 15-30 ml within a $24 \mathrm{~h}$ period (Kerns et al., 2000; Krinke, 2000). Thus, urinary leakage throughout the day was likely in both injury groups, because the VRA denervated the EUS, causing the muscle to lose its tone, become flaccid, and atrophy (Pavlakis et al., 1983). Thus, any pressure over the abdominal area may overcome the urethral resistance and cause urine to leak out. This confounding factor limits the interpretability of the urine collection data as an outcome measure for continence in rodent lower motor neuron injury models. Thus, our findings highlight the importance of performing urodynamic studies to differentiate the ability of implanted rats to initiate reflex bladder contractions, activate the EUS, and actively void.

\section{Reinnervation of the LUT function after injury}

A variety of surgical strategies using nerve root anastomosis have been undertaken in attempts to achieve reinnervation of the LUT 

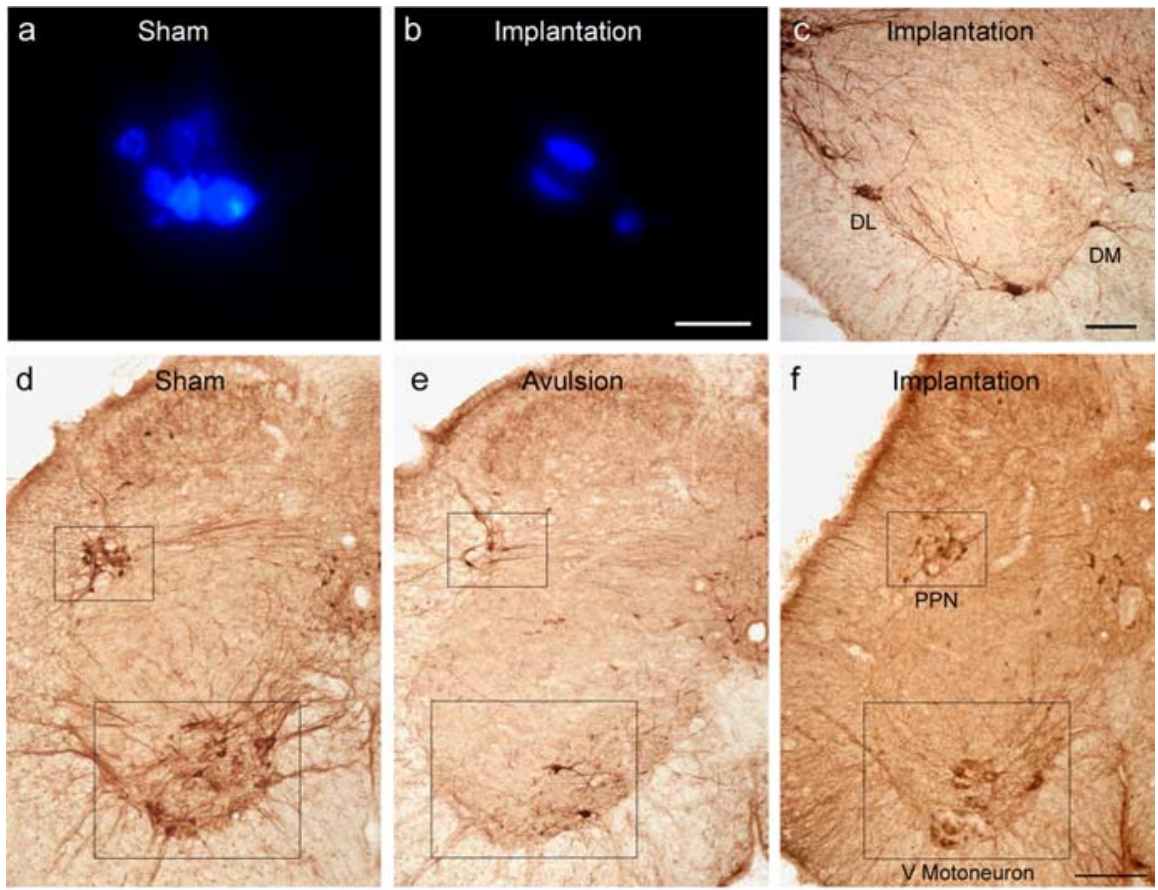

g
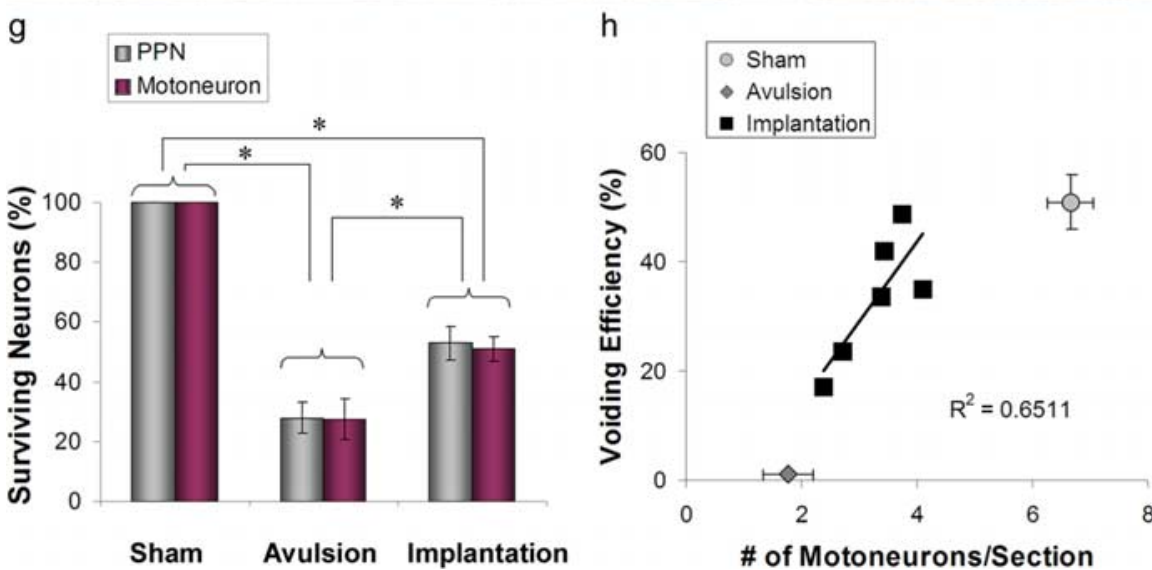

Figure 5. Morphological analysis shows EUS reinnervation by DL motoneurons and neuroprotection of the spinal cord efferent neurons at 12 weeks after operation. $\boldsymbol{a}, \boldsymbol{b}$, Fast Blue retrogradely labeled DL motoneurons in the $L 6$ segment are detected only in the sham $(\boldsymbol{a})$ and implanted $(\boldsymbol{b})$ groups after tracer injections into the EUS muscle. $\boldsymbol{c} \boldsymbol{- \boldsymbol { f }}$, Spinal cord sections were processed for ChAT to identify PPNs and somatic motoneurons in sham (d), avulsed (e), and implanted $(\boldsymbol{c}, \boldsymbol{f})$ groups. The L6 segment (c) includes the DL and DM nuclei, whereas the $S 1$ segment $(\boldsymbol{d}-\boldsymbol{f})$ contains the majority of PPNs and ventral motoneurons. $\boldsymbol{g}$, Stereological counts demonstrate a significant avulsion-induced death of PPNs and motoneurons. After implantation, there is a significant increase in the percentage of surviving PPNs and motoneurons compared with after avulsion but still a significant decrease compared with sham. PPN and motoneuronal survival are not significantly different from each other after avulsion or implantation. $\boldsymbol{h}$, The number of surviving motoneurons is significantly correlated to voiding efficiency in the implanted group. Both graphs represent all three motor nuclei $(\mathrm{V}, \mathrm{DL}$, and DM) combined. Each filled square represents one animal in the implanted group, whereas animals in the sham and avulsed group are represented as mean \pm SE. ${ }^{*} p<0.05$. Scale bars: (in $\left.\boldsymbol{b}\right) \boldsymbol{a}, \boldsymbol{b}, 100 \mu \mathrm{m} ; \boldsymbol{c}, 200$ $\mu \mathrm{m}$; (in $\boldsymbol{f}) \boldsymbol{d}-\boldsymbol{f}, 300 \mu \mathrm{m}$.

Table 2. Number of surviving neurons in the L6 and S1 segments at 12 weeks after operation

\begin{tabular}{lccc}
\hline & Sham $(n=6)$ & Avulsed $(n=4)$ & Implanted $(n=6)$ \\
\hline PPN & $725 \pm 40$ & $194 \pm 31^{*}$ & $383 \pm 58^{* *}$ \\
V & $273 \pm 17$ & $92 \pm 21^{*}$ & $163 \pm 13^{* *}$ \\
DL & $77 \pm 10$ & $5 \pm 2^{*}$ & $21 \pm 4^{* *}$ \\
DM & $45 \pm 6$ & $12 \pm 5^{*}$ & $17 \pm 2^{* *}$ \\
\hline
\end{tabular}

Stereological counts of ChAT-immunolabeled PPNs and V motoneurons spanning $1.6 \mathrm{~mm}$ of the $\mathrm{S} 1$ segment as well as DL and DM motoneurons spanning $1 \mathrm{~mm}$ of the $\mathrm{L} 6$ segment. ${ }^{*} p<0.05$ compared with both sham and implanted groups; ${ }^{* *} p<0.05$ compared with sham. after injury. End-to-end anastomosis of injured ventral roots to adjacent intact ventral roots has resulted in bladder improvements in rats (Xiao and Godec, 1994), cats (Carlsson and Sundin, 1968; Vorstman et al., 1986; Xiao et al., 1999), canines (Ruggieri et al., 2004), and humans (Carlsson and Sundin, 1980; Xiao et al., 2003). This procedure requires an additional lesion to uninjured neurons and a shift in function by the originally uninjured neurons to now reinnervate a new target. Furthermore, the intact dorsal roots create a new somatic-CNS-bladder reflex circuitry, whereby scratching the skin in specific dermatomes may elicit bladder contractions (Xiao et al., 1999, 2003). Although nerve-to-nerve anastomosis requires peripheral nerve regeneration, our repair model differs in that CNS regeneration is also necessary for reestablishment of the micturition circuitry by the originally injured neurons without inflicting any additional lesions to uninjured neurons.

Our implantation procedure nearly doubled PPN and motoneuronal survivals at 3 months after operation, similar to the rescue effects reported in our unilateral VRA and root implantation model at 6 weeks (Hoang et al., 2006). A lessened neuroprotective effect, but not axonal reinnervation by surviving neurons, may be expected with delayed implantations, because previous studies have shown that only motoneuronal survival declined with increased delay of nerve implantation after avulsion (Wu et al., 2004). It is of interest to note that long-term neuroprotective effects by root implantations into the spinal cord are controversial, because some investigators have shown no motoneuronal protection at 3-4 months (Bergerot et al., 2004; Blits et al., 2004), whereas others have demonstrated a sustained improvement of motoneuronal survival at both 12 and 20 weeks after a cervical root implantation (Gu et al., 2004). Although we show a significant increase in cell survival, not all axotomized neurons were protected. Therefore, it is not necessarily surprising that a complete return of normal bladder function was not observed. Furthermore, VRA injuries have been shown to activate synaptic stripping (Novikov et al., 2000), an elimination of presynaptic terminals from the cell body and dendrites of axotomized neurons (Sumner, 1975; Oliveira et al., 2004). Thus, although the surviving autonomic and motor neurons may be able to reinnervate peripheral targets, the synaptic inputs to those neurons may have undergone reorganizational changes. However, information on such possible synaptic plasticity in the conus medullaris is sparse.

In the implanted series, reinnervation of the LUT was evident by the functional bladder and EUS recordings, decrease in blad- 
der size and weight, and neuronal labeling in the spinal cord after retrograde tracer injections into the EUS muscle. After VRA injury, unlike the denervated EUS muscle that becomes flaccid, the bladder maintains innervation by the postganglionic parasympathetic neurons. Thus, in avulsed rats, the bladders were hypertrophied rather than atrophied, similar to the findings in rats after a midthoracic injury (Kruse et al., 1993; Pikov and Wrathall, 2001; Mitsui et al., 2005). However, after implantation, the bladder size was normalized and was associated with the re-establishment of reflexive micturition. It is also interesting to note that despite our previous findings that a single implanted root can attract regenerating axons of motoneurons from multiple adjacent segments (Hoang and Havton, 2006b), there was a high degree of reinnervation specificity by axotomized motoneurons, because no retrogradely labeled neurons were detected outside of the DL motor pool in the implanted series. One possible mechanism may involve synaptic competition, in which the synaptic connections of some regenerating motoraxons survive and proliferate, whereas others innervating the same peripheral target are eliminated at the neuromuscular junction (Lichtman and Colman, 2000). Such synaptic elimination has been shown to occur by 8 weeks after a sciatic nerve injury in adult rats (McArdle, 1975; Gorio et al., 1983). Thus, by 12 weeks after implantation, we speculate that neurons that incorrectly reached the sphincter muscle may have retracted their axons.

\section{Activation of the detrusor and EUS muscles}

In the adult rat, normal micturition requires simultaneous activation of both the striated EUS and the smooth detrusor muscle (de Groat, 2006). Pharmacological inhibition of the EUS with the preserved function of the detrusor is sufficient to achieve some voiding. However, the bursting activity of the EUS is necessary for complete emptying of the rat bladder (Maggi et al., 1986; Mersdorf et al., 1993; Kakizaki et al., 1997). Interestingly, our results show a positive correlation between voiding efficiency and the number of surviving motoneurons but not PPNs in the implanted series, emphasizing the role and importance of functional innervation of striated sphincter and pelvic muscles by somatic motoneurons for efficient voiding. However, in the implanted rats, the frequently continuous rather than bursting activity of the EUS may diminish the efficiency of peristaltic urine outflow. Furthermore, EUS activation was not absolutely essential for micturition to occur, because some voiding still transpired in the absence of EUS activation if the bladder contractions were sufficiently strong.

The rise in bladder pressure during contractions was decreased in the implanted rats compared with sham rats, and in contrast to findings after midthoracic injuries (Cheng and de Groat, 2004), indicating that not as much force was produced and necessary to initiate voiding, probably because of a reduced sphincter tone. In addition, the presence of nonvoiding contractions suggests that the spino-bulbo-spinal reflex was activated by bladder distension, but not enough muscle fibers were recruited to expel the urine. Alternatively, nonvoiding contractions may be attributable to an increased urethral resistance, but this scenario is more likely after midthoracic injuries (Kruse et al., 1993; Pikov et al., 1998). Although synchronous activation of the EUS was present during bladder contractions in both the sham and implanted groups, in some animals of the latter group, the EUS EMG activation was delayed and prolonged relative to the duration of the bladder contraction. We speculate that this might be caused by a disturbance in the intramedullary activation of a feedback loop system. Occasional EMG activation without blad- der contractions or bladder contractions without EMG activation occurred, also suggesting that the normal intramedullary neural circuitry and communication between autonomic and motor neurons were not fully re-established. Altogether, these findings may explain why the implanted rats were able to void less efficiently than sham rats.

\section{Conclusion}

We demonstrated in a rat cauda equina injury and repair model that an acute implantation of avulsed lumbosacral ventral roots into the conus medullaris results in functional and anatomical reinnervation of the LUT with marked improvement in voiding efficiency. We propose that, for select cases of traumatic conus medullaris/cauda equina injuries, future treatments may include the use of surgical implantation of lesioned lumbosacral ventral roots to provide a conduit for autonomic and somatic motor neurons to extend axons from the spinal cord to the peripheral nervous system and to promote functional recovery of the LUT.

\section{References}

Akhavan M, Hoang TX, Havton LA (2006) Improved detection of fluorogold-labeled neurons in long-term studies. J Neurosci Methods 152:156-162.

Anderson KD (2004) Targeting recovery: priorities of the spinal cordinjured population. J Neurotrauma 21:1371-1383.

Barber RP, Phelps PE, Houser CR, Crawford GD, Salvaterra PM, Vaughn JE (1984) The morphology and distribution of neurons containing choline acetyltransferase in the adult rat spinal cord: an immunocytochemical study. J Comp Neurol 229:329-346.

Bergerot A, Shortland PJ, Anand P, Hunt SP, Carlstedt T (2004) Cotreatment with riluzole and GDNF is necessary for functional recovery after ventral root avulsion injury. Exp Neurol 187:359-366.

Beric A, Light JK (1992) Function of the conus medullaris and cauda equina in the early period following spinal cord injury and the relationship to recovery of detrusor function. J Urol 148:1845-1848.

Blits B, Carlstedt TP, Ruitenberg MJ, de Winter F, Hermens WT, Dijkhuizen PA, Claasens JW, Eggers R, van der Sluis R, Tenenbaum L, Boer GJ, Verhaagen J (2004) Rescue and sprouting of motoneurons following ventral root avulsion and reimplantation combined with intraspinal adeno-associated viral vector-mediated expression of glial cell linederived neurotrophic factor or brain-derived neurotrophic factor. Exp Neurol 189:303-316.

Carlsson CA, Sundin T (1968) Reconstruction of severed ventral roots innervating the urinary bladder. An experimental study in cats. Scand J Urol Nephrol 2:199-210.

Carlsson CA, Sundin T (1980) Reconstruction of afferent and efferent nervous pathways to the urinary bladder in two paraplegic patients. Spine 5:37-41.

Carlstedt T, Lind H, Cullheim S, Risling M (1986) Reinnervation of hindlimb muscles after ventral root avulsion and implantation in the lumbar spinal cord in the adult rat. Acta Physiol Scand 128:645-646.

Carlstedt T, Grane P, Hallin RG, Noren G (1995) Return of function after spinal cord implantation of avulsed spinal nerve roots. Lancet 346 : $1323-1325$

Chai H, Wu W, So KF, Yip HK (2000) Survival and regeneration of motoneurons in adult rats by reimplantation of ventral root following spinal root avulsion. Neuroreport 11:1249-1252.

Cheng CL, de Groat WC (2004) The role of capsaicin-sensitive afferent fibers in the lower urinary tract dysfunction induced by chronic spinal cord injury in rats. Exp Neurol 187:445-454.

Coggeshall RE, Lekan HA (1996) Methods for determining numbers of cells and synapses: a case for more uniform standards of review. J Comp Neurol 364:6-15.

de Groat WC (1998) Anatomy of the central neural pathways controlling the lower urinary tract. Eur Urol 34:2-5.

de Groat WC (2006) Integrative control of the lower urinary tract: preclinical perspective. Br J Pharmacol 147:S25-40.

Fowler CJ (1999) Neurological disorders of micturition and their treatment. Brain 122:1213-1231.

Fowler CJ, Kirby RS, Harrison MJ, Milroy EJ, Turner-Warwick R (1984) 
Individual motor unit analysis in the diagnosis of disorders of urethral sphincter innervation. J Neurol Neurosurg Psychiatry 47:637-641.

Gorio A, Carmignoto G, Finesso M, Polato P, Nunzi MG (1983) Muscle reinnervation. II. Sprouting, synapse formation and repression. Neuroscience 8:403-416.

Gu HY, Chai H, Zhang JY, Yao ZB, Zhou LH, Wong WM, Bruce I, Wu WT (2004) Survival, regeneration and functional recovery of motoneurons in adult rats by reimplantation of ventral root following spinal root avulsion. Eur J Neurosci 19:2123-2131.

Hallin RG, Carlstedt T, Nilsson-Remahl I, Risling M (1999) Spinal cord implantation of avulsed ventral roots in primates; correlation between restored motor function and morphology. Exp Brain Res 124:304-310.

Hoang TX, Havton LA (2006a) Novel repair strategies to restore bladder function following cauda equina/conus medullaris injuries. Prog Brain Res 152:195-204.

Hoang TX, Havton LA (2006b) A single re-implanted ventral root exerts neurotropic effects over multiple spinal cord segments in the adult rat. Exp Brain Res 169:208-217.

Hoang TX, Nieto JH, Tillakaratne NJ, Havton LA (2003) Autonomic and motor neuron death is progressive and parallel in a lumbosacral ventral root avulsion model of cauda equina injury. J Comp Neurol 467:477-486.

Hoang TX, Nieto JH, Dobkin BH, Tillakaratne NJ, Havton LA (2006) Acute implantation of an avulsed lumbosacral ventral root into the rat conus medullaris promotes neuroprotection and graft reinnervation by autonomic and motor neurons. Neuroscience 138:1149-1160.

Kakizaki H, de Groat WC (1996) Role of spinal nitric oxide in the facilitation of the micturition reflex by bladder irritation. J Urol 155:355-360.

Kakizaki H, Fraser MO, De Groat WC (1997) Reflex pathways controlling urethral striated and smooth muscle function in the male rat. Am J Physiol 272:R1647-R1656.

Keirstead HS, Fedulov V, Cloutier F, Steward O, Duel BP (2005) A noninvasive ultrasonographic method to evaluate bladder function recovery in spinal cord injured rats. Exp Neurol 194:120-127.

Kerns JM, Damaser MS, Kane JM, Sakamoto K, Benson JT, Shott S, Brubaker L (2000) Effects of pudendal nerve injury in the female rat. Neurourol Urodyn 19:53-69.

Krinke GJ (2000) The laboratory rat, Ed 1. London: Academic.

Kruse MN, Belton AL, de Groat WC (1993) Changes in bladder and external urethral sphincter function after spinal cord injury in the rat. Am J Physiol 264:R1157-R1163.

Lichtman JW, Colman H (2000) Synapse elimination and indelible memory. Neuron 25:269-278.

Maggi CA, Giuliani S, Santicioli P, Meli A (1986) Analysis of factors involved in determining urinary bladder voiding cycle in urethananesthetized rats. Am J Physiol 251:R250-R257.

Marson L (1997) Identification of central nervous system neurons that innervate the bladder body, bladder base, or external urethral sphincter of female rats: a transneuronal tracing study using pseudorabies virus. J Comp Neurol 389:584-602.

Matsuura S, Downie JW (2000) Effect of anesthetics on reflex micturition in the chronic cannula-implanted rat. Neurourol Urodyn 19:87-99.

McArdle JJ (1975) Complex end-plate potentials at the regenerating neuromuscular junction of the rat. Exp Neurol 49:629-638.

McKenna KE, Nadelhaft I (1986) The organization of the pudendal nerve in the male and female rat. J Comp Neurol 248:532-549.

Mersdorf A, Schmidt RA, Tanagho EA (1993) Urodynamic evaluation and electrical and pharmacologic neurostimulation. The rat model. Urol Res 21:199-209.

Mitsui T, Shumsky JS, Lepore AC, Murray M, Fischer I (2005) Transplantation of neuronal and glial restricted precursors into contused spinal cord improves bladder and motor functions, decreases thermal hypersensitivity, and modifies intraspinal circuitry. J Neurosci 25:9624-9636.

Nadelhaft I, Booth AM (1984) The location and morphology of pregangli- onic neurons and the distribution of visceral afferents from the rat pelvic nerve: a horseradish peroxidase study. J Comp Neurol 226:238-245.

Nieto JH, Hoang TX, Warner EA, Franchini BT, Westerlund U, Havton LA (2005) Titanium mesh implantation-a method to stabilize the spine and protect the spinal cord following a multilevel laminectomy in the adult rat. J Neurosci Methods 147:1-7.

Novikov LN, Novikova LN, Holmberg P, Kellerth J (2000) Exogenous brain-derived neurotrophic factor regulates the synaptic composition of axonally lesioned and normal adult rat motoneurons. Neuroscience 100:171-181.

Oliveira AL, Thams S, Lidman O, Piehl F, Hokfelt T, Karre K, Linda H, Cullheim S (2004) A role for MHC class I molecules in synaptic plasticity and regeneration of neurons after axotomy. Proc Natl Acad Sci USA 101:17843-17848.

Pavlakis AJ, Siroly MB, Goldstein I (1983) Neurologic findings in conus medullaris and cauda equina injury. Arch Neurol 40:570-573.

Pikov V, Wrathall JR (2001) Coordination of the bladder detrusor and the external urethral sphincter in a rat model of spinal cord injury: effect of injury severity. J Neurosci 21:559-569.

Pikov V, Gillis RA, Jasmin L, Wrathall JR (1998) Assessment of lower urinary tract functional deficit in rats with contusive spinal cord injury. J Neurotrauma 15:375-386.

Potter PJ (2006) Disordered control of the urinary bladder after human spinal cord injury: what are the problems? Prog Brain Res 152:51-57.

Rende M, Giambanco I, Buratta M, Tonali P (1995) Axotomy induces a different modulation of both low-affinity nerve growth factor receptor and choline acetyltransferase between adult rat spinal and brainstem motoneurons. J Comp Neurol 363:249-263.

Ruggieri MR, Barbe M, Braverman AS, Andrea LD, Kozin SH, Pontari MA, Betz RR (2004) Reinnervation of the canine urinary bladder by somatic nerve transfer. Soc Neurosci Abstr 30:310.11.

Schalow G, Bersch U, Michel D, Koch HG (1995) Detrusor-sphincteric dyssynergia in humans with spinal cord lesions may be caused by a loss of stable phase relations between and within oscillatory firing neuronal networks of the sacral micturition center. J Auton Nerv Syst 52:181-202.

Schroder HD (1980) Organization of the motoneurons innervating the pelvic muscles of the male rat. J Comp Neurol 192:567-587.

Shefchyk SJ (2002) Spinal cord neural organization controlling the urinary bladder and striated sphincter. Prog Brain Res 137:71-82.

Sumner BE (1975) A quantitative analysis of boutons with different types of synapse in normal and injured hypoglossal nuclei. Exp Neurol 49:406-417.

Vorstman B, Schlossberg SM, Kass L, Devine Jr CJ (1986) Urinary bladder reinnervation. J Urol 136:964-969.

West MJ (1999) Stereological methods for estimating the total number of neurons and synapses: issues of precision and bias. Trends Neurosci 22:51-61.

Wu W, Chai H, Zhang J, Gu H, Xie Y, Zhou L (2004) Delayed implantation of a peripheral nerve graft reduces motoneuron survival but does not affect regeneration following spinal root avulsion in adult rats. J Neurotrauma 21:1050-1058.

Xiao CG, Godec CJ (1994) A possible new reflex pathway for micturition after spinal cord injury. Paraplegia 32:300-307.

Xiao CG, de Groat WC, Godec CJ, Dai C, Xiao Q (1999) "Skin-CNSbladder" reflex pathway for micturition after spinal cord injury and its underlying mechanisms. J Urol 162:936-942.

Xiao CG, Du MX, Dai C, Li B, Nitti VW, de Groat WC (2003) An artificial somatic-central nervous system-autonomic reflex pathway for controllable micturition after spinal cord injury: preliminary results in 15 patients. J Urol 170:1237-1241.

Yoshiyama M, Roppolo JR, De Groat WC (1994) Alteration by urethane of glutamatergic control of micturition. Eur J Pharmacol 264:417-425. 